\title{
Analysis of the Activity-Deprived Zebrafish Mutant macho Reveals an Essential Requirement of Neuronal Activity for the Development of a Fine-Grained Visuotopic Map
}

\author{
Lara Gnuegge, ${ }^{1}$ Susanne Schmid, ${ }^{2}$ and Stephan C. F. Neuhauss ${ }^{1}$ \\ ${ }^{1}$ Max-Planck-Institut für Entwicklungsbiologie, 72076 Tübingen, Germany, and 2Zoologisches Institut, Universität \\ Tübingen, 72076 Tübingen, Germany
}

The formation of a retinotopic map is thought to involve an activity-independent molecular phase for early steps of both axon pathfinding and projection and a later phase in which cross talk between retinal ganglion cells (RGCs) and tectal neurons modifies and refines the neuronal connections. We report that the maturation of the retinotopic map in the zebrafish tectum involves activity-dependent processes. Zebrafish larvae mutant for the gene macho (mao) lack neuronal activity in RGCs and also display an enlarged retinotectal projection field but no significant increase in single axon length. This morphological defect can be phenocopied by raising larvae under TTX-induced neural impulse blockade. The effect of activity deprivation is dependent on the developmental stage. The projection phenotype in mao as well as in the TTX-treated

The projection of retinal ganglion cells (RGCs) to the tectum in all lower vertebrates is topographically organized, whereby the map of RGC arbors on the tectum reflects the inverted map of RGC bodies in the retina. The precise pattern of neuronal connectivity is established during the development of the nervous system by the concerted work of two broad mechanisms. The first one involves molecular cues of target recognition and occurs before the neurons become functionally active. This process leads to a topographically correct map that is refined in a second phase by the emerging patterns of neuronal activity (Meyer, 1982; Schmidt and Edwards, 1983; Cline and Constantine-Paton, 1989; Goodman and Shatz, 1993). The final accuracy of connections determines the animal's ability to see and resolve fine details of the visual world.

The zebrafish retinotectal map develops in the early larval stages as a topographically organized map (Burrill and Easter, 1994). The first axons of retinal ganglion cells leave the eye at 34-36 hr post-fertilization (hpf) through the optic stalk. They grow toward the midline in two fascicles of either dorsal or ventral origin and cross at the optic chiasm. Growth cones of RGC axons then invade the contralateral tectum at $46-48 \mathrm{hpf}$ and project to

\footnotetext{
Received April 13, 2000; revised March 1, 2001; accepted March 6, 2001.

We thank S. A. Holley and J. M. Rick for their critical reading of this manuscript and O. Biehlmaier for assistance in the analysis of retinal morphology. We are grateful to F. Bonhoeffer for his support of this work. We thank the anonymous reviewers for valuable comments.

Correspondence should be addressed to Stephan Neuhauss at his present address: Brain Research Institute, Department of Neuromorphology, Eidgenössische Technische Hochschule Zürich, Winterthurerstrasse 190, CH-8057 Zürich, Switzerland. E-mail: neuhauss@hifo.unizh.ch.

L. Gnuegge's present address: University of Freiburg, Zoology I, Hauptstrasse 1, 79104 Freiburg, Germany.

Copyright (C) 2001 Society for Neuroscience $\quad 0270-6474 / 01 / 213542-07 \$ 15.00 / 0$
}

larvae develops between 4 and 6 d post-fertilization (dpf), after complete tectal coverage is first achieved. Electrophysiological recordings of RGCs in wild-type and mao zebrafish larvae reveal a temporally regulated reduction of sodium current in the mutant between 5 and $6 \mathrm{dpf}$. This coincides with the time of the axonal projection shifting on the tectum to compensate for the disparate growth patterns of the retina and the tectum. Our genetic and physiological analyses suggest a model in which neuronal activity in RGCs is needed for the establishment of morphological plasticity.

Key words: visual system; retinotectal projection; sodium currents; zebrafish; axon guidance; plasticity; Danio rerio; retinal ganglion cell; TTX

their topographically appropriate site. During larval development and also throughout adult life of the zebrafish, new ganglion cells continue to differentiate at the retinal periphery, sending axons to their target area in the tectum (Marcus et al., 1999). To compensate for the disparate pattern of cell addition (circumferentially in the retina and caudomedially in the tectum), the first topographic map must refine to accommodate the later ingrowing axons during the maturation of the retinotectal projection.

Initial experiments, in which the role of neuronal activity in this process of refinement and shifting were investigated, indicate that neuronal activity in RGCs is not necessary in the early development of the retinotectal projection. Blockade of action potentials by application of tetrodotoxin (TTX) to zebrafish larvae between 2 and 4 d post-fertilization (dpf) did not lead to alterations in arbor fields on the tectum (Stuermer et al., 1990), as has been reported previously for regenerating axons in TTXtreated goldfish tectum (Schmidt and Edwards, 1983).

Using the zebrafish mutant macho (mao), we take a genetic approach to address the question of activity-dependent fine mapping in the retinotectal system of the zebrafish. mao belongs to a group of retinotectal mutations, identified in the large scale Tübingen screen (Baier et al., 1996; Karlstrom et al., 1996; Trowe et al., 1996). mao mutant larvae display enlarged arborization fields and lack visually guided behavior (Neuhauss et al., 1999). In electrophysiological studies, we demonstrated that RGCs in these mutants are incapable of firing action potentials. By blocking sodium channels with TTX up to $6 \mathrm{dpf}$, we can phenocopy the retinotectal defects found in the mutant. In combination with results from single axon labelings, we show that activity plays a role in the maturation of the retinotectal system during the period of disparate growth of the retina and the tectum. 


\section{MATERIALS AND METHODS}

Fish maintenance. Zebrafish were reared and crossed as described previously (Haffter et al., 1996a). Embryos were kept at $28^{\circ} \mathrm{C}$ in $\mathrm{E} 3$ medium (in mM: $5 \mathrm{NaCl}, 0.17 \mathrm{KCl}, 0.33 \mathrm{CaCl}_{2}$, and $0.33 \mathrm{MgSO}_{4}$ ) in the presence of $150 \mu \mathrm{m}$ of 1-phenyl-2-thiourea (Sigma, Deisenhofen, Germany) to prevent pigment formation (Westerfield, 1994). macho (mao ${ }^{\mathrm{tt} 261}$ ) embryos were obtained from matings of identified heterozygous carriers. The mao mutation is recessive, and homozygous embryos were identified on the basis of their lack of touch response (Granato et al., 1996). Controls consisted of unaffected sibling embryos. Heterozygous larvae were indistinguishable from wild-type (wt) larvae.

TTX injections. Larvae between 2 and $5 \mathrm{dpf}$ received injections of 8-10 $\mathrm{nl}$ of 0.5-0.9 mu TTX (Calbiochem, Darmstadt, Germany) in Ringer's solution in one eye or into the tectal region of the midbrain, delivered with a pointed glass needle by pressure as described previously (Stuermer et al., 1990). After successful injections, larvae are entirely paralyzed, except for their heartbeat. We used paralysis as an indicator of successful drug application.

Labeling of RGCs. Dye injections were performed as described previously (Baier et al., 1996). Zebrafish larvae were fixed in $4 \%$ paraformaldehyde for a minimum of $12 \mathrm{hr}$ at room temperature and then mounted in agarose $(1.2 \%$ in PBS, $30 \%$ in water) to label projections fields of groups of RGCs with 1,1'-dioctadecyl-3,3,3',3'-tetramethylindocarbocyanine perchlorate (DiI) (Molecular Probes, Leiden, The Netherlands).

For single axon labeling, live larvae were anesthetized in $0.02 \%$ 3-aminobenzoic acid methyl ester (Sigma) and mounted on a wet tissue paper. A minimum amount of DiI solution (half saturated in ethanol) was injected with a Pneumatic PicoPump PV820 (World Precision Instruments, Sarasota, FL) into the nasodorsal retina to label axons in the posterior tectum. After an incubation time of $12 \mathrm{hr}$, the larvae were fixed and mounted dorsal side down on a petriPERM dish (hydrophilic; Heraeus, Hanau, Germany). Images of labeled axons were digitally captured on a Leica (Nussloch, Germany) inverted confocal microscope. The size of the tectal lobes was visualized by the absence of nuclear staining with 4,6-diamidino-2-phenylindole (DAPI) $(0.2 \mu \mathrm{g} / \mathrm{ml}$; Sigma) on the tectal neuropil. Image analysis was performed with the objectimage software (NIH Image software) and later transferred to an Excel (MicroSoft, Seattle, WA) worksheet for statistic evaluations. Retinal ganglion cell axon morphology and length were analyzed by reconstructing the confocal image overlay and the scale bar onto an acetate sheet, to scan in and further analyze it. The total branch length was measured by redrawing the axon from the first branching point to the very tip of the axon (including the main branch) and comparing it with the scale bar with the object-image analysis software. Total branch length was used, because distinction between main and side branch was often not clear-cut because of the branching pattern. For analyzing the tectal coverage, branch tips of each axon were connected, and the thereby outlined area was calculated again by the object-image analysis software.

Histology. Fixed larvae were dehydrated in a graded series of ethanolwater mixtures incubated in 1:1 ethanol $99.9 \%$ and Technovit 7100 basic solution (Heraeus) for $2 \mathrm{hr}$. After overnight infiltration in Technovit 7100 basic solution, larvae were positioned in Technovit 7100 polymerization medium for $2 \mathrm{hr}\left(37^{\circ} \mathrm{C}\right)$.

Microtome sections $(3 \mu \mathrm{m})$ were prepared and mounted on poly-Llysine-coated slides. Sections were then air dried at $60^{\circ} \mathrm{C}$, stained with Richardson solution (1\% azur, 1\% methylenblue, and 1\% Borax in $\mathrm{ddH}_{2} \mathrm{O}$ ), overlaid with DPX mounting medium (Agar Scientific Ltd., Stansted, UK), and coverslipped.

Electrophysiological recordings. Zebrafish were transferred into extracellular solution (ec) (in mm: $150 \mathrm{NaCl}, 3 \mathrm{KCl}, 2 \mathrm{CaCl}_{2}, 1 \mathrm{MgCl}_{2}, 10$ HEPES, and 10 glucose, $\mathrm{pH} 7.5$ adjusted with $1 \mathrm{M} \mathrm{NaOH}$ ) and immobilized by $100 \mu \mathrm{M}$ D-tubocurarine. After decapitation, one eye was removed, and the retina was isolated by the use of two fine, electrically sharpened tungsten wires. For electrophysiological recordings, retinas were transferred into a poly-L-lysine-coated recording chamber on a microscope (Axioskop; Zeiss, Oberkochen, Germany) and fixed (inside up) with a small grid made of fine nylon strings tightened between an U-shaped platinum wire. The recording chamber was superfused with $1.5-2 \mathrm{ml}$ of oxygenated ec per minute. Voltage-clamp experiments were performed in the perforated-patch mode. Patch pipettes were pulled out of borosilicate capillaries (Biologica). Pipette resistance was 4-10 $\mathrm{M} \Omega$ after heat polishing.

For perforated-patch recordings, pipettes first were frontfilled with a pipette solution (in mM: $90 \mathrm{Cs}$-acetate, $40 \mathrm{CsCl}_{2}, 1 \mathrm{MgCl}_{2}, 0.2 \mathrm{CaCl}_{2}, 10$ EGTA, and 10 HEPES, pH 7.2 adjusted with $1 \mathrm{M} \mathrm{CsOH}$; all from Sigma) and thereafter backfilled with the same solution plus gramicidin in a concentration of $20 \mu \mathrm{g} / 5 \mathrm{ml}$ (Sigma). After sealing, light suction was applied, until some minutes later when the series resistance had decreased to a constant level at $\sim 20 \mathrm{M} \Omega$. Cell capacity and series resistance was then compensated, and recordings were started.

For the recording of action potentials in current-clamp mode, the pipette solution consisted of (in $\mathrm{mM}$ ): $135 \mathrm{KCl}, 10 \mathrm{HEPES}$, and 10 EGTA, pH 7.2 with $\mathrm{KOH}$. The bath solution contained (in mM): 145 $\mathrm{NaCl}, 3 \mathrm{KCl}, 10 \mathrm{CaCl}_{2}$, and 10 HEPES, pH 7.2 with $\mathrm{NaOH}$. Resting membrane potential was determined as the membrane voltage measured in current clamp with no applied current. For the recording of action potentials, the initial holding potential was kept near $-80 \mathrm{mV}$ by steadystate injection of current. Action potentials were elicited by applying a series of depolarizing current pulses $(200 \mathrm{msec})$ of increasing amplitude, ranging between 10 and $100 \mathrm{pA}$ by steps of $10 \mathrm{pA}$.

All recordings were made with an Axopatch 200 A amplifier (Axon Instruments, Foster City, CA) at a sampling rate of $5 \mathrm{kHz}$. Series resistance compensation was usually $60-80 \%$. The liquid junction potential was $6.8 \mathrm{mV}$, and the voltage-clamp data were corrected accordingly. TTX was applied by a fast computer-driven pressure application system (ALA Scientific Instruments, Westbury, NY). The commercial software program pClamp 8 (Axon Instruments) was used for data acquisition and analysis. Data were displayed and stored for subsequent offline analysis on an IBM computer.

Eye movement recordings. Larvae were put into a Petri dish (diameter, $3.5 \mathrm{~cm}$ ) containing $2.5 \%$ methylcellulose $\left(28^{\circ} \mathrm{C}\right)$ in E3 medium to partially immobilize the animals. Immobilization helps suppress the optomotor response and permits easy scoring of eye movements. To allow optimal viewing conditions, larvae were positioned dorsal side up with the help of a dissecting needle. The dish was placed inside a rotating drum (diameter, $5 \mathrm{~cm}$ ) fitted with black and white stripes (eight black stripes of $23^{\circ}$ width). The drum was illuminated by white light from below and rotated at $4-12 \% \mathrm{sec}$. Optokinetic responses were elicited by clockwise and counterclockwise rotation.

\section{RESULTS}

In the zebrafish, as well as in other vertebrates with nonoverlapping visual fields, RGC axons project exclusively to the contralateral tectum, in which they branch in a topographic manner.

The genetic analysis of zebrafish mutants provides a new approach to the understanding of the development of the vertebrate visual system. Several genes required for pathfinding projection were found in the Tübingen screen for retinotectal projection defects. Larvae mutant for one of those genes, mao, show projection defects on the tectum (Trowe et al., 1996). mao mutant larvae also exhibit defects in mechanosensation, rendering the larvae touch insensitive (Granato et al., 1996). Mutant larvae fail to develop a swim bladder, like many other mutations found during the screen, and die between 7 and 8 dpf. Because embryonic development occurs externally and zebrafish larvae are completely translucent, the tectal region in embryos and young larvae can be observed easily under a light microscope.

Examination of the visually guided behavior of homozygous mao mutants revealed a defect in the optokinetic response (Neuhauss et al., 1999). The combined results of the visual behavior and the defect in the projection pattern of RGC axons on the optic tectum led us to investigate the role of neuronal activity in the development of the retinotectal projection in the mao mutant. To visualize the axonal projection on the tectum, we used anterograde labeling by lipophilic tracer dyes. Dye crystals were placed into the retina by using a setup that was designed for high throughput dye injections (Baier et al., 1996). With this procedure, the amount of dye and the point of injection are highly reproducible. Further analysis of the projection defect included single axon labelings and electrophysiological recordings.

\section{Retinotectal projection defects in mao larvae}

The earliest stage at which RGC axon terminals can be seen on the tectum is at $44 \mathrm{hpf}$. New RGCs continue to differentiate and 

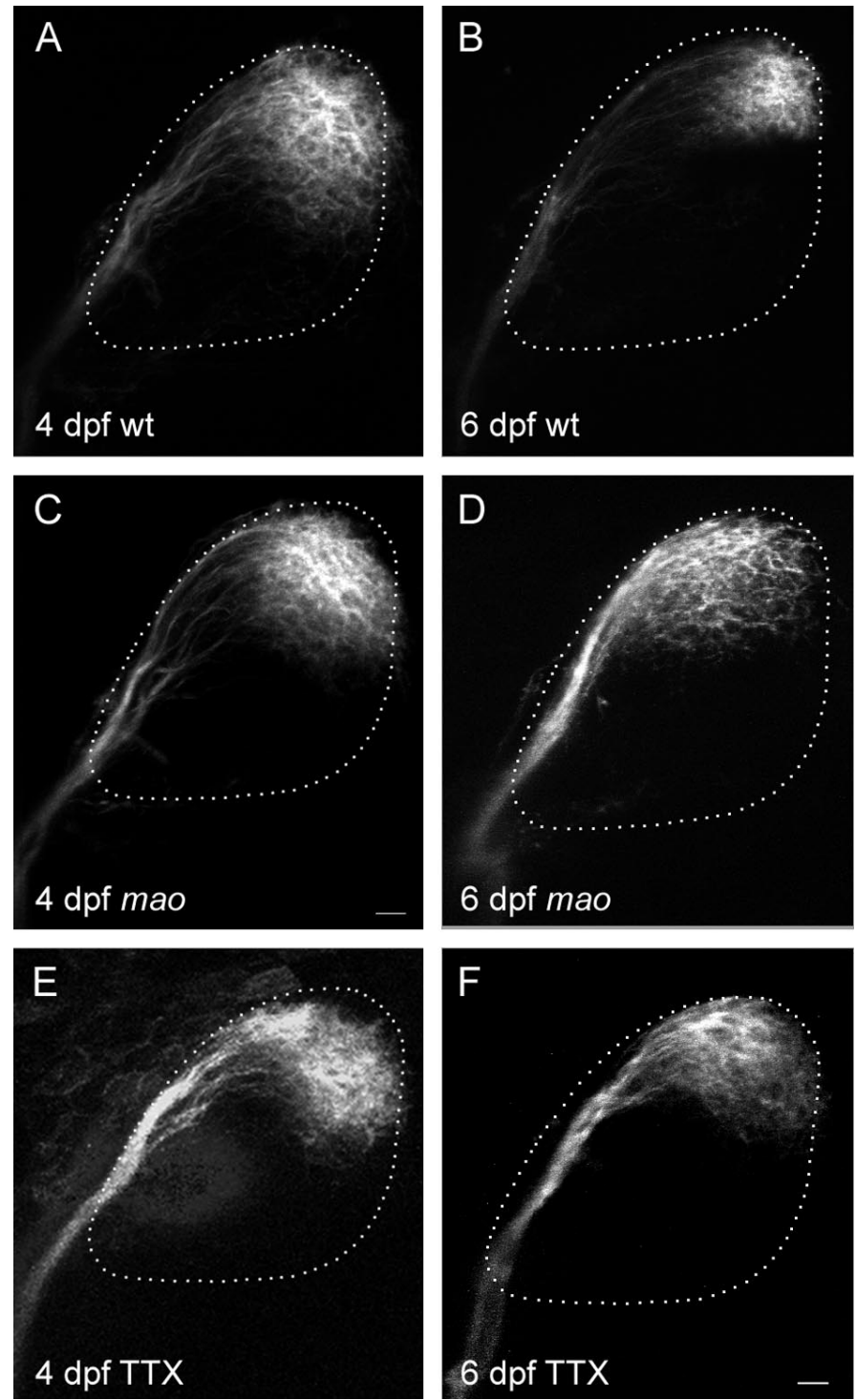

Figure 1. Retinotectal projection of nasodorsal RGCs in the mao mutant and in TTX-treated larvae is normal at $4 \mathrm{dpf}$ but enlarged at $6 \mathrm{dpf}$. Dorsal view, anterior to the left. Retinal ganglion cells are labeled by injection of DiI into the nasodorsal quadrant of the retina. The size of the tectal neuropil is outlined with a dashed line. $A$, Wild-type projection at $4 \mathrm{dpf}$ cover $\sim 40 \%$ of the tectum. $B$, Nasodorsal axons of $6 \mathrm{dpf}$ wild-type larvae terminate in the posterior lateral tectum, covering approximately onefourth of the total tectum. $C$, The projection of nasodorsal RGCs in mao mutant larvae at $4 \mathrm{dpf}$ resembles the wild-type projection at this age. $D$, Projection in mao at $6 \mathrm{dpf}$. The termination area of nasodorsal axons is larger and more dispersed than in the wild type. $E$, The projection size in TTX-treated larvae at $4 \mathrm{dpf}$ is not altered compared with the wt projection. $F$, At $6 \mathrm{dpf}$, TTX treatment led to an increase in the projection field, resembling the projection in mao larvae of the same developmental stage. Scale bar, $10 \mu \mathrm{m}$.

send axons to the tectum throughout larval and adult life. The earliest defects in the retinotectal system of mao mutant larvae are visible at $5 \mathrm{dpf}$. This coincides with the time that complete coverage of the tectum by RGC axons is achieved. Labeling of RGC axons in mutant larvae at $4 \mathrm{dpf}$ does not reveal any distinction from wild type in the projection pattern (Fig. 1C).

The projection of RGC axons onto the tectum in mao mutant larvae at $6 \mathrm{dpf}$ is topographic but less well defined than in wild type (Trowe et al., 1996) (Fig. 1D). The order in the optic tract of mutant axons after leaving the eye through the optic stalk and pathfinding toward the tectum appears to be normal. However, a group of axons originating in the nasodorsal quadrant of the mutant retina cover a tectal area that extends the normal projection field by $\sim 30 \%(p<0.01)$, whereas the size of tecta in each group of larvae does not vary significantly. Curiously, in three of 22 labeled tecta, axons from the nasodorsal half of the retina continue to grow along the posterior side of the tectum and terminate in the medial instead of the lateral posterior quadrant of the tectum. This phenomenon can also be observed in some wild-type strains, other than the Tübingen strain, used as control in our experiments. Projection fields of tempoventral RGCs appear more condensed in the mutant. Because the axon terminals in the anterior part of the tectum normally display a denser topography, differences in the projection of temporal RGC axons are difficult to determine. Therefore, we have concentrated our analysis on the posterior tectum.

\section{mao larvae exhibit visual impairment}

The mutation in the mao gene not only affects the morphology of RGC axons on the tectum but also the function of the visual system. When mao mutant larvae (identified by the inability to respond to a touch stimulus) are presented with a moving grating of black and white stripes, only a fraction of animals shows the normal behavior of an optokinetic nystagmus (OKN): following the stripes as they move across the larva's visual field and snapping back in a fast saccade. In wt larvae, this behavior starts to develop at $3 \mathrm{dpf}$ and is fully mature at $5 \mathrm{dpf}$ (Easter and Nicola, 1996). In a developmental series, in which the visual behavior was observed on 4 consecutive days between 4 and $7 \mathrm{dpf}$, individual mutant larvae show no significant change in visual response. Different degrees of impairment in larvae with a reduced touch response are found in clutches from different heterozygous parents. Only $40 \%(n=70)$ of the mutants (isolated on the basis of the missing touch response) execute a normal OKN. In most of the mutant larvae, the saccades are either executed only once or twice $(30 \%)$, or the response is completely missing (30\%), regardless of the developmental stage, although spontaneous eye movements can frequently be observed. Mutant larvae with a reduced or absent OKN also have a very intense melanophore phenotype, whereas melanophores in mutants with a normal visual behavior appear smaller. The impaired visual behavior in combination with the lack of response to a brightening of the background by melanophore contraction indicates that mao mutants have a reduction in light perception (Neuhauss et al., 1999).

\section{Analysis of the retinal morphology}

Defects in visual behavior in vertebrates can have several possible causes. The disruption of any step in the cascade of visual information processing could lead to a defective optokinetic response. To localize the defect in mao mutant larvae, we examined the retina of mutant larvae for morphological defects.

Histological sections through the eyes of wild-type and mutant 6-d-old larvae revealed no morphological defect in the retina of the mao mutation (data not shown). The retina is normally layered with the pigment epithelium surrounding the outer retina. In close contact with the pigment epithelium are the outer segments of the photoreceptors. They are normal in shape and size. No apparent sign of degeneration in these cells or the outer retina can be observed, consistent with an unaffected response to light measured by electroretinography (ERG) (Neuhauss et al., 1999). Because activity of RGCs cannot be measured in the ERG, we 
examined the functionality of this cell type by other means. Considering the sodium conductance defect in primary sensory neurons in mao (Ribera and Nusslein-Volhard, 1998), an attractive possibility to explain the lack of visually evoked behavior in mao is a reduction in neuronal activity in RGCs.

\section{Influence of TTX on the development of the retinotectal projection}

A lack of action potentials in RGCs could be responsible for the impairment in visual behavior, as well as the enlarged projection field of the RGC axons. The dispersed projection field of the nasodorsal RGC axons seen in the mao mutation is highly reminiscent of the arborization pattern of adult goldfish RGCs, grown under the influence of the sodium channel blocker TTX (Meyer, 1983). This prevents the neurons from firing action potentials and renders the fish blind. Axons from regenerating RGCs grown under the influence of TTX display enlarged projection fields (Meyer, 1983).

To test whether the dispersed projection in the mutant is linked to a lack of neuronal activity, we injected the sodium channel blocker TTX into the tectum or the eye of zebrafish larvae at 2-4 $\mathrm{dpf}$ and reared them for another $2 \mathrm{~d}$. In zebrafish larvae, local application of TTX is not possible because the drug diffuses freely throughout the body, blocking all TTX-sensitive sodium channels, including sodium channels in motor neurons. The resulting paralysis of the larvae was used as an internal control for drug delivery. The persistence of the activity blockage is monitored through the absence of the larva's swimming behavior. Larvae that were still paralyzed at the end of this period were fixed, and the retinotectal projection was labeled with DiI.

When 2-d-old zebrafish are injected with TTX and the projection develops without neuronal activity until day 4 , no effects on the arborization pattern of the labeled projection can be observed. The posterior lateral quadrant of the tecta of both control and TTX-treated larvae are covered with RGC-axon branches (Fig. 1E). The size of the labeled tectal area appears larger than in older animals, reflecting the higher number of RGCs labeled at $4 \mathrm{dpf}$ because of the relatively large needle. These results are consistent with earlier work, in which activity blockage is reported to have no effect on single axon morphology in the zebrafish larvae treated from 2 to $4 \mathrm{dpf}$ with TTX (Stuermer et al., 1990).

In neither 4-d-old mao larvae nor activity-deprived wild-type fish of this age is the pattern of axonal arborization disturbed (Fig. 1C,E). Because we observed the first defects in the mao retinotectal projection at $5 \mathrm{dpf}$, we proceeded to test the influence of TTX on later stages of the visual system development. When larvae are reared from day 4 to day 6 under the influence of the sodium channel blocker, we see an enlargement of the projection field of RGC axons with nasal origin by $11 \%(p<0.1)$, rather similar to the mao projection phenotype at $6 \mathrm{dpf}$ (Fig. $1 D, F$ ). This indicates that lack of activity has no effect until day 4 of development and shows an effect at $6 \mathrm{dpf}$. These results are consistent with the results found for the mao mutation, in which the defect also can be seen at $6 \mathrm{dpf}$ but not at $4 \mathrm{dpf}$.

\section{Single axon morphology in mao and TTX-treated larvae}

The finding that TTX treatment leads to an enlargement of retinotectal projection fields allows at least two possible explanations. The dispersed projection could be attributable to an increase in branch number, branch size, or the relative position of
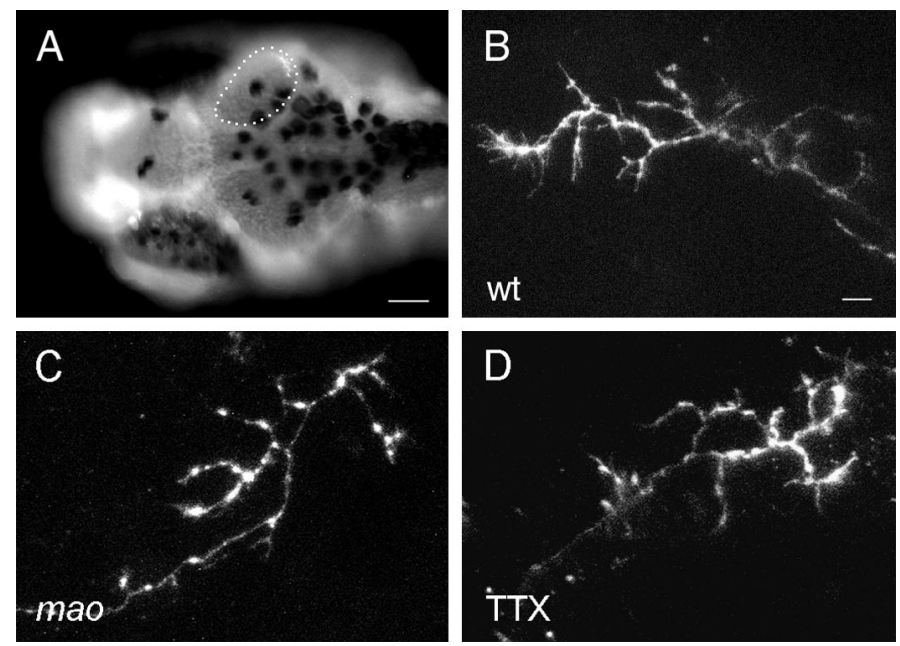

Figure 2. Morphology of individual RGC axons is not altered in mao mutant or TTX-treated larval tecta at $6 \mathrm{dpf}$. $A$, DAPI-stained tectal neuropil of a wild-type larva with a single axon terminal labeled with DiI in the posterior medial quadrant. Branching patterns of single RGC axons in wild-type $(B)$, mao mutants $(C)$, and TTX-treated larvae $(D)$ visualized by DiI injection into the retina. Scale bar: $A, 50 \mu \mathrm{m} ; B-D, 10 \mu \mathrm{m}$.

Table 1. Variations in length, branch number, and tectal coverage, reflecting the influence of neuronal activity on single axon morphology

\begin{tabular}{lccc} 
& wt $(n=14)$ & mao $(n=12)$ & TTX $(n=11)$ \\
\hline Total branch length & & & \\
$\quad(\mu \mathrm{m})$ & $195.5 \pm 154.5$ & $125.7 \pm 53.0$ & $171.5 \pm 124.3$ \\
Branch number & $12.4 \pm 7.4$ & $11.9 \pm 4.8$ & $13.0 \pm 5.2$ \\
$\begin{array}{l}\text { Tectal coverage } \\
\left(\mu \mathrm{m}^{2}\right)\end{array}$ & $447.3 \pm 337.9$ & $691.9 \pm 456.5$ & $543.9 \pm 494.5$ \\
$\begin{array}{l}\text { Length/branch } \\
\quad(\mu \mathrm{m})\end{array}$ & $15.4 \pm 5.4$ & $11.3 \pm 5.4$ & $14.7 \pm 7.4$
\end{tabular}

$\overline{\text { Average branch length of individual axons is decreased in mao mutants compared }}$ with wt (ANOVA, single-factor analysis; $p<0.05$ ). The same trend is observed in TTX-injected activity-deprived larvae. Branch number and total branch length do not differ significantly.

one axon toward the axon of a neighboring RGC. Thus, we decided to investigate the size and distribution of single axons. For this purpose, a minimal amount of DiI solution was injected into the nasal part of the retina to label single axon terminals in the posterior tectum.

The branching pattern of axons in wild-type, mutant, and TTX-treated larvae is very similar (Fig. 2). Axons from the periphery branch mainly into the direction of the tectal center, whereas more centrally located axons show no preference for any branching direction. However, it seems that the branching pattern in mao axons is less directed toward the leading direction of the main branch, rendering the branching area larger than in wt axons. The number of branches within one group varies considerably (in wt, between 4 and 29), possibly indicating that the labeled group of ganglion cells are composed of different ganglion cell types.

Comparison of labeled axons from wild-type, mao, and TTXtreated larvae nevertheless point out differences (Table 1). Measurements of these single axons show a decrease in average branch length in mao compared with wt $(11.3 \pm 5.4 \mu \mathrm{m} ; p=$ 0.05). Comparison between TTX-treated and wild-type larvae branch lengths does not yield statistically significant differences. 


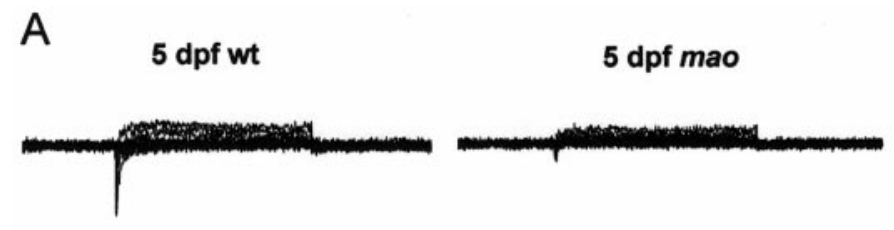

B
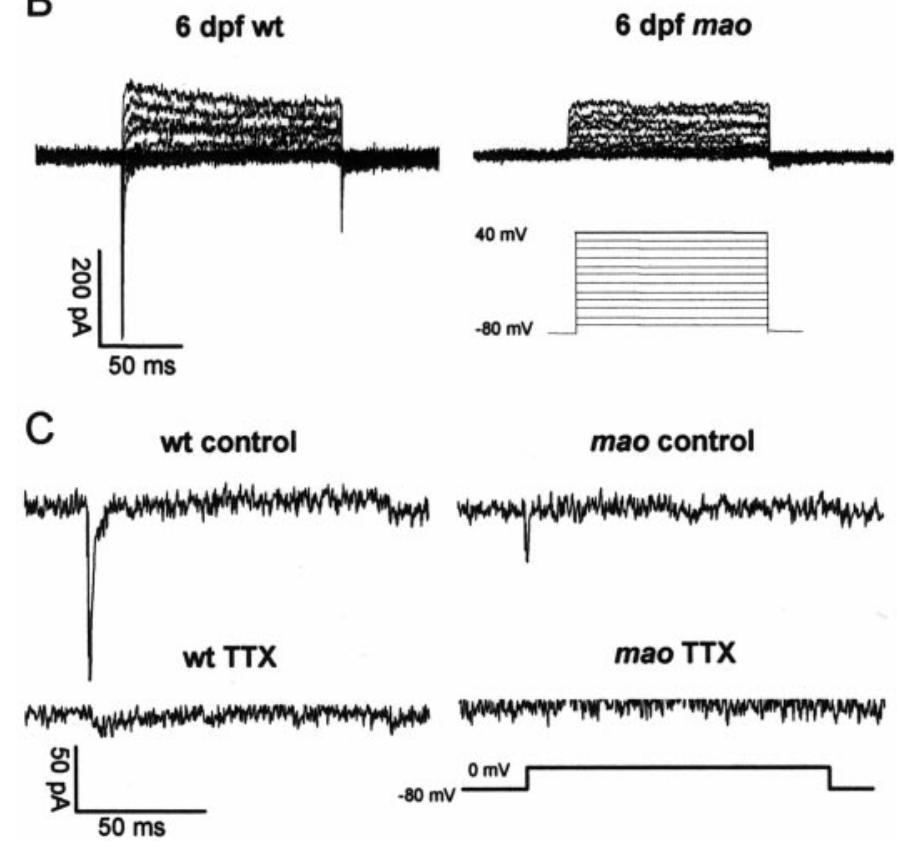

Figure 3. Original registrations of voltage-clamp recordings of wild-type and mutant (mao) RGCs from 5 and $6 \mathrm{dpf}$. $A$, RGC cell membranes were held at $-80 \mathrm{mV}$, and increasingly depolarizing voltage steps were applied. Current traces of $5 \mathrm{dpf}$ wild-type RGCs reveal larger transient inward and larger sustained outward currents than of $5 \mathrm{dpf}$ mutant RGCs. B, Current traces of $6 \mathrm{dpf}$ wild-type and mutant RGCs. The same protocol and scaling was used as in $A$. Current amplitudes of $6 \mathrm{dpf}$ wild-type RGCs are larger than in $5 \mathrm{dpf}$ RGCs. The sustained outward current in $6 \mathrm{dpf}$ mutant RGCs is also larger than in $5 \mathrm{dpf}$ mutant RGCs, whereas the transient inward current has completely disappeared. $C$, Application of $5 \mu \mathrm{M}$ TTX completely blocks the transient inward current in both wild-type and mutant RGC. This indicates that the transient inward current is exclusively driven by sodium influx through voltage-activated sodium channels.

These data indicate that the observed enlargement of the arborization field in activity-deprived RGC axons cannot be accounted for by increased tectal coverage of individual axons.

\section{mao RGCs have reduced sodium currents}

The similarity in retinotectal projection defects in mao mutants and TTX-treated larvae suggests a defect in neuronal activity in the RGCs being responsible for the enlarged projection fields. We established a whole-mount preparation for patch-clamp recordings from zebrafish RGCs to examine the ion channel activity in detail. In this preparation, the RGC layer comes to lie on the exposed side of the retina and is therefore easily accessible with a patch-clamp electrode. Voltage- and current-clamp recordings of RGCs were performed in the perforated-patch and wholecell mode.

In voltage-clamp recordings, the membrane potential was held at $-80 \mathrm{mV}$, and increasingly depolarizing pulses of $120 \mathrm{msec}$ duration and in $10 \mathrm{mV}$ steps were applied. Current traces of mao and wild-type RGCs are shown for 5 and $6 \mathrm{dpf}$ in Figure 3, $A$ and
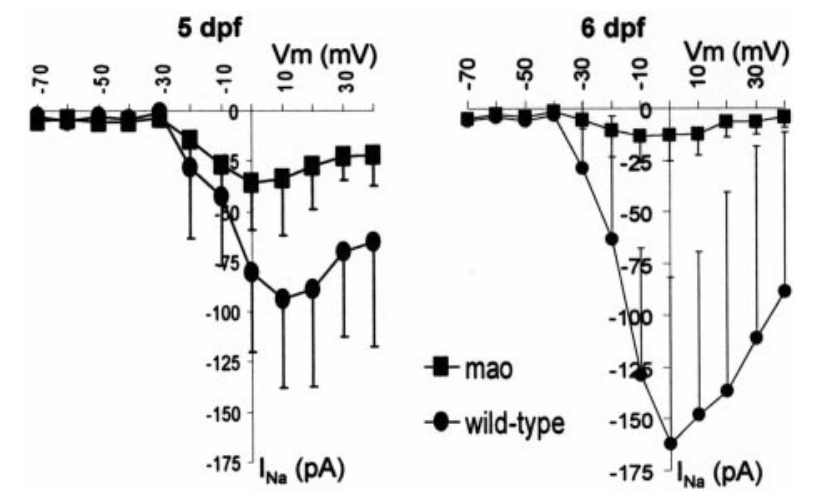

Figure 4. Current-voltage relationship of the TTX-sensitive inward current in wild-type and mutant RGCs at 5 and 6 dpf. Mean values with SD for each group are plotted. The large SD results from differences in absolute amplitudes between individual RGCs. The inward current amplitude in mao mutant larvae show a significant decrease from 5 to $6 \mathrm{dpf}$, whereas wild-type sodium current amplitude increases at the same time. For $n$ and values at $0 \mathrm{mV}$, see Results.

$B$. In wild-type RGCs, depolarizing pulses elicited large transient inward currents, followed by sustained outward currents. The amplitude of both current types increased between 5 and 6 dpf. In mutant RGCs, only the sustained outward current increased, whereas the transient inward current decreased during this time period. Application of TTX showed that the transient inward currents were always completely blocked by this agent, indicating that this current type is mediated by sodium influx through TTX-sensitive voltage-activated sodium channels (Fig. 3C). In Figure 4, the mean current-voltage relationship ( $I-V$ plot) of the sodium current is plotted for mutant and wild-type RGCs of 5 and 6 , dpf respectively. At a membrane potential of $0 \mathrm{mV}$, wild-type RGCs show an increase of transient inward current amplitude from $-81 \pm 39$ (mean $\pm \mathrm{SD} ; n=7$ ) to $-162 \pm 81$ (mean $\pm \mathrm{SD} ; n=9$ ) pA between 5 and $6 \mathrm{dpf}$. At $5 \mathrm{dpf}$, sodium current amplitude is already reduced in mutant RGCs to $44 \%$ of the current in wild-type RGCs. The mean amplitude in mutant $\mathrm{RGCs}$ at $0 \mathrm{mV}$ is $-36 \pm 23 \mathrm{pA}$ (mean $\pm \mathrm{SD} ; n=10$ ). At $6 \mathrm{dpf}$, current density in mutant RGCs decreases further to $-13 \pm 12$ $\mathrm{pA}$ (mean $\pm \mathrm{SD} ; n=7$ ) or $8 \%$ of the wild-type values (Fig. 4 ). Thus, there is a developmental downregulation of functional voltage-gated sodium channels in mao RGCs from dpf 5 onward. Accordingly, current-clamp recordings revealed that RGCs of mao mutant embryos at $6 \mathrm{dpf}$ are no longer able to generate action potentials in response to the injection of small depolarizing currents. This is shown in Figure 5, in which voltage-clamp recordings and current-clamp recordings are shown for the same wild-type (left) and mutant (right) RGC.

\section{DISCUSSION}

Analysis of the zebrafish mutant mao has revealed a defect in neuronal activity in RGCs that leads to a dispersed projection pattern of the axon terminals on the tectum. Mutant larvae display a lack of visual behavior, and morphological analysis of the retinotectal projection revealed a defect in the mapping of RGC axon terminals on the tectum. In electrophysiological recordings, a pronounced reduction of sodium currents resulting in the inability to generate action potentials could be demonstrated in mutant RGCs. Pharmacological experiments in which sodium currents were suppressed by the application of TTX gave a retinotectal phenotype similar to the one found in the mao 

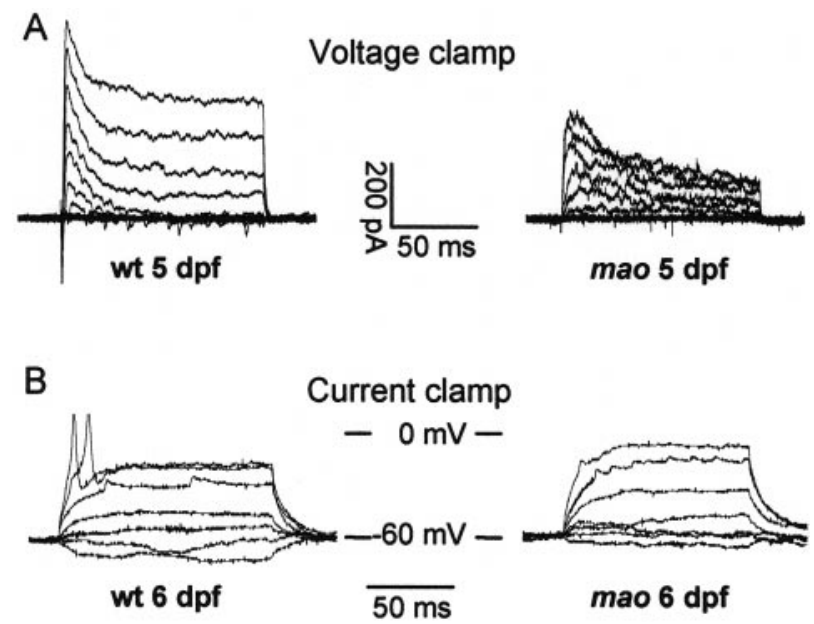

Figure 5. Whole-cell voltage-clamp $(A)$ and current-clamp $(B)$ recordings of retinal ganglion cells in flat-mount retinas of mao mutant larvae at $6 \mathrm{dpf}$. Voltage-clamp recordings reveal a lack of the TTX-sensitive transient inward current in mao RGCs $(A)$. Under current-clamp conditions, the same cell fails to fire overshooting action potentials $(B)$. For voltage and current protocols, see Materials and Methods.

mutant. Thus, we conclude that the dispersed projection in mao larvae is attributable to a lack of action potentials in RGCs.

A role for neuronal activity in the development of the topographic projection in the retinotectal system had been proposed previously (Archer et al., 1982; O'Leary et al., 1986; Sretavan et al., 1988; Cook et al., 1999). However, results from experiments in which TTX was used to block neuronal activity in the visual system of developing zebrafish suggested no significant role for neuronal activity in the mapping process (Stuermer et al., 1990). In these experiments, TTX was injected into the eyes of 1.5-d-old larvae. Injection of $\mathrm{DiI}$ to label RGC arbors on the tectum was executed at 3-4 dpf. The labeling of terminal arbors of the nasodorsal RGC axon group and of individual terminal arbors shows that the precision of the retinal axon terminal order is not perturbed by neural impulse blockade during this early period of development.

In our experiments, we examined possible later effects of neuronal activity on the maturation of the retinotectal projection. The morphological phenotype in mao and TTX-treated larvae is only visible from $5 \mathrm{dpf}$ onward. The occurrence of this defect at a relatively late stage in the development of the retinotectal projection leads to the conclusion that neuronal activity is necessary for the stabilization but not the establishment of the topographic map on the tectum. The influence of activity-dependent processes in the stabilization and the refinement of the projection has been described for other vertebrates. Experiments with Rana pipiens have shown that axons from a transplanted third eye project to both tecta, in which they segregate into eye-specific stripes (Constantine-Paton and Law, 1978). This segregation happens via pruning of axonal branches to limit the projection to a defined area. Treatment with TTX blocks this segregation procedure, and individual arbors are larger than in the striped tectum (Reh and Constantine-Paton, 1985). Related studies demonstrated that treatment of Xenopus RGCs with the NMDA receptor blocker APV resulted in an increased rate of axonal branch tip addition and retraction but had no effect on arbor morphology (Rajan et al., 1999). This indicates a transient rather than permanent change in axon morphology, which cannot be detected in fixed preparations, but would still lead to a change in the overall distribution of axons on the tectum. This may be part of the reason why we did not observe a more dramatic effect of activity deprivation on single axon morphology, because we used fixed developmental time points as opposed to a time-lapse analysis.

The dispersed appearance of projection fields in mao and TTX-treated axons can be attributed to the inhibition of cross talk between tectal neurons and RGCs, which then leads to a destabilization of RGC axon terminals. This means that the determination of neighborhood relationships depends on the ability of the neurons to fire action potentials. These can be evoked by either visual input or, even before the opening of the eyes, spontaneous waves of activity that cross the retina within the RGC layer (Wong et al., 1993). Raising zebrafish larvae in the dark does not influence the development of the retinotectal projection (L. Gnuegge, unpublished observation). Therefore, spontaneous activity seems to be the relevant activity for plasticity in the lateral geniculate nucleus or the tectum, respectively. Morphological plasticity becomes especially relevant in the maturation of the visuotopic map. In teleosts, the retina progressively adds new cells at the periphery, such that cell differentiation proceeds in a central to peripheral direction (Marcus et al., 1999). However, the tectal lobes have a gradient of cell proliferation and maturation that proceeds from rostrolateral to caudomedial (Nguyen et al., 1999). Because the retina projects to the tectum in a topographic manner while the two structures are still adding new cells throughout life, the retinal projection must continually shift. RGC axon terminals have to migrate from rostral tectal positions to successively more caudal positions as the tectum matures to accommodate the disparate patterns of growth (Gaze et al., 1974; Easter and Stuermer, 1984). All of these developmental processes occur in an animal with a fully functional visual system. Experimental evidence for this shifting hypothesis comes from studies in Rana pipiens, in which the relationship between ganglion cells from near the optic nerve head and a centrally located group of labeled tectal neurons was studied for the duration of larval life (Reh and Constantine-Paton, 1984). A growing distance between the RGC terminals and the patch of labeled tectal neurons gives evidence for the existence of shifting RGC arbor terminals. A defect in this process would lead to a phenotype that we observe in the mao mutation. Central RGC axons do not shift posterior with a caudally extending tectum, but new RGC axons from the nasal periphery of the eye still project to the now larger caudal part of the tectum. The termination field of the nasal half of RGC axons will appear enlarged. This apparent enlargement is attributable to a stretching of the projection in an anterior to posterior direction. Therefore, the distribution of single axon terminals on the tectum gives rise to a dispersed projection. Axons from neighboring RGCs in the retina would stabilize their synapses onto the same tectal neuron by firing at approximately the same time. If neuronal activity is disturbed, the correlated firing is inhibited and neighborhood relationships cannot be determined. As a result, synapses are not stabilized and the overlap of axon terminals from neighboring RGCs decreases.

The relative position of axon terminals on the tectum is difficult to deduce from single axon analysis. Experiments in which TTX was used to block action potentials early in the development of the retinotectal projection did not lead to an increase in tectal coverage of single axons, although blocking voltage-gated potassium channels leads to a reduction of axon growth (McFarlane and Pollock, 2000), a variable that was not included in the TTX 
experiments. Considering these results, the decrease in average branch length that we detected in our experiments is unlikely to account for the overall projection defect found in the mao mutant larvae at $6 \mathrm{dpf}$ (Stuermer et al., 1990).

In the electrophysiological analysis of mutant larvae, a progressive loss of sodium conductance in RGCs from 5 to $6 \mathrm{dpf}$ is measured, although sodium conductance increases in wild-type cells. These divergent results suggest the involvement of two sequential functions, of which only the later acting one is affected in the mao mutant. Whereas the early component, which seems to be downregulated by $6 \mathrm{dpf}$, is unaffected in mao, the later appearing molecule is upregulated in wild type, taking over the function of the early component. Progressive downregulation of the early component therefore would explain the decrease of sodium conductance in mao RGCs from 5 to $6 \mathrm{dpf}$. Developmentally regulated genes that act sequentially in the establishment of a functionally active visual system are likely to account for the defect in sodium conductance in the mao mutant. Possible candidates would be regulatory genes required for the upregulation of the later sodium current or components of the sodium channel itself. At least four different sodium channel $\alpha$ subunits are known to be expressed in the rat retina (Fjell et al., 1997). In several neuronal cell types, these and other channel subunits have been shown to be expressed in spatially and temporally diverse patterns (Felts et al., 1997). A sodium channel $\alpha$ subunit would therefore be a good candidate to be affected by the mao mutation.

The behavioral defect in mao, however, is not developmentally regulated. Larvae with reduced touch response either show a normal $\mathrm{OKN}$ at 4 and $7 \mathrm{dpf}$ or they display a reduced or missing OKN. Therefore, the observed variance in visual behavior is likely attributable to an incomplete inactivation of the mao gene by the mutation.

The data presented here provides novel evidence for a role of neuronal activity in the maturation of the retinotopic projection in the young zebrafish. Lack of neural impulse activity in ganglion cells leads to a defect in the shifting of axon terminals as a response to the disparate growth pattern of retina and tectum and results in a dispersed projection. This result indicates that the zebrafish can be used as a model organism for studying mechanisms of morphological plasticity. Moreover, the availability of mutants affecting neuronal activity in several subclasses of neurons might be an additional attraction to study the development of neuronal connections in this vertebrate.

\section{REFERENCES}

Archer SM, Dubin MW, Stark LA (1982) Abnormal development of kitten retino-geniculate connectivity in the absence of action potentials. Science 217:743-745.

Baier H, Klostermann S, Trowe T, Karlstrom RO, Nusslein-Volhard C, Bonhoeffer F (1996) Genetic dissection of the retinotectal projection. Development 123:415-425.

Burrill JD, Easter Jr SS (1994) Development of the retinofugal projections in the embryonic and larval zebrafish (Brachydanio rerio). J Comp Neurol 346:583-600.

Cline HT, Constantine-Paton M (1989) NMDA receptor antagonists disrupt the retinotectal topographic map. Neuron 3:413-426.

Constantine-Paton M, Law MI (1978) Eye-specific termination bands in tecta of three-eyed frogs. Science 202:639-641.

Cook PM, Prusky G, Ramoa AS (1999) The role of spontaneous retinal activity before eye opening in the maturation of form and function in the retinogeniculate pathway of the ferret. Vis Neurosci 16:491-501.

Easter Jr SS, Nicola GN (1996) The development of vision in the zebrafish (Danio rerio). Dev Biol 180:646-663.
Easter Jr SS, Stuermer CA (1984) An evaluation of the hypothesis of shifting terminals in goldfish optic tectum. J Neurosci 4:1052-1063.

Felts PA, Yokoyama S, Dib-Hajj S, Black JA, Waxman SG (1997) Sodium channel alpha-subunit mRNAs I, II, III, NaG, Na6 and hNE (PN1): different expression patterns in developing rat nervous system. Brain Res Mol Brain Res 45:71-82.

Fjell J, Dib-Hajj S, Fried K, Black JA, Waxman SG (1997) Differential expression of sodium channel genes in retinal ganglion cells. Brain Res Mol Brain Res 50:197-204.

Gaze RM, Keating MJ, Chung SH (1974) The evolution of the retinotectal map during development in Xenopus. Proc R Soc Lond B Biol Sci 185:301-330.

Goodman CS, Shatz CJ (1993) Developmental mechanisms that generate precise patterns of neuronal connectivity. Cell [Suppl] 72:77-98.

Granato M, van Eeden FJ, Schach U, Trowe T, Brand M, Furutani-Seiki M, Haffter P, Hammerschmidt M, Heisenberg CP, Jiang YJ, Kane DA, Kelsh RN, Mullins MC, Odenthal J, Nusslein-Volhard C (1996) Genes controlling and mediating locomotion behavior of the zebrafish embryo and larva. Development 123:399-413.

Haffter P, Granato M, Brand M, Mullins MC, Hammerschmidt M, Kane DA, Odenthal J, van Eeden FJ, Jiang YJ, Heisenberg CP, Kelsh RN, Furutani-Seiki M, Vogelsang E, Beuchle D, Schach U, Fabian C, Nusslein-Volhard C (1996a) The identification of genes with unique and essential functions in the development of the zebrafish, Danio rerio. Development 123:1-36.

Karlstrom RO, Trowe T, Klostermann S, Baier H, Brand M, Crawford AD, Grunewald B, Haffter P, Hoffmann H, Meyer SU, Muller BK, Richter S, van Eeden FJ, Nusslein-Volhard C, Bonhoeffer F (1996) Zebrafish mutations affecting retinotectal axon pathfinding. Development 123:427-438.

Marcus RC, Delaney CL, Easter Jr SS (1999) Neurogenesis in the visual system of embryonic and adult zebrafish (Danio rerio). off. Vis Neurosci 16:417-424.

McFarlane S, Pollock NS (2000) A role for voltage-gated potassium channels in the outgrowth of retinal axons in the developing visual system. J Neurosci 20:1020-1029.

Meyer RL (1982) Tetrodotoxin blocks the formation of ocular dominance columns in goldfish. Science 218:589-591.

Meyer RL (1983) Tetrodotoxin inhibits the formation of refined retinotopography in goldfish. Brain Res 282:293-298.

Neuhauss SC, Biehlmaier O, Seeliger MW, Das T, Kohler K, Harris WA, Baier H (1999) Genetic disorders of vision revealed by a behavioral screen of 400 essential loci in zebrafish. J Neurosci 19:8603-8615.

Nguyen V, Deschet K, Henrich T, Godet E, Joly JS, Wittbrodt J, Chourrout D, Bourrat F (1999) Morphogenesis of the optic tectum in the medaka (Oryzias latipes): a morphological and molecular study, with special emphasis on cell proliferation. J Comp Neurol 413:385-404.

O'Leary DD, Fawcett JW, Cowan WM (1986) Topographic targeting errors in the retinocollicular projection and their elimination by selective ganglion cell death. J Neurosci 6:3692-3705.

Rajan I, Witte S, Cline HT (1999) NMDA receptor activity stabilizes presynaptic retinotectal axons and postsynaptic optic tectal cell dendrites in vivo. J Neurobiol 38:357-368.

Reh TA, Constantine-Paton M (1984) Retinal ganglion cell terminals change their projection sites during larval development of Rana pipiens. J Neurosci 4:442-457.

Reh TA, Constantine-Paton M (1985) Eye-specific segregation requires neural activity in three-eyed Rana pipiens. J Neurosci 5:1132-1143.

Ribera AB, Nusslein-Volhard C (1998) Zebrafish touch-insensitive mutants reveal an essential role for the developmental regulation of sodium current. J Neurosci 18:9181-9191.

Schmidt JT, Edwards DL (1983) Activity sharpens the map during the regeneration of the retinotectal projection in goldfish. Brain Res 269:29-39.

Sretavan DW, Shatz CJ, Stryker MP (1988) Modification of retinal ganglion cell axon morphology by prenatal infusion of tetrodotoxin. Nature 336:468-471.

Stuermer CA, Rohrer B, Munz H (1990) Development of the retinotectal projection in zebrafish embryos under TTX-induced neural-impulse blockade. J Neurosci 10:3615-3626.

Trowe T, Klostermann S, Baier H, Granato M, Crawford AD, Grunewald B, Hoffmann H, Karlstrom RO, Meyer SU, Muller B, Richter S, Nusslein-Volhard C, Bonhoeffer F (1996) Mutations disrupting the ordering and topographic mapping of axons in the retinotectal projection of the zebrafish, Danio rerio. Development 123:439-450.

Westerfield M (1994) The zebrafish book, Ed 2.1. Eugene, OR: University of Oregon.

Wong RO, Meister M, Shatz CJ (1993) Transient period of correlated bursting activity during development of the mammalian retina. Neuron 11:923-938. 